\title{
Mécanismes de déformation dans les argiles surconsolidées
}

M. HATTAB

Laboratoire de mécanique des sols, structures et matériaux

École centrale de Paris Grande Voie des Vignes 92295 Châtenay-Malabry

Cedex

mahdia.hattab@ecp.fr

\section{P.-Y. HICHER}

Institut de recherche en génie civil et mécanique GeM-UMR CNRS 6183

École centrale de Nantes BP 92101

44321 Nantes Cedex 3
L'objectif de cette étude est la caractérisation expérimentale des mécanismes de déformation dans une argile surconsolidée (kaolinite P300) sur chemin triaxial axisymétrique. Un intérêt particulier est porté au mécanisme déviatoire dû à la partie déviatoire du tenseur des contraintes. La représentation dans l'espace (e, p', q) des résultats d'essais sur chemins purement déviatoires et l'utilisation du modèle Cam-clay permettent de suivre P'évolution du chemin expérimental et de voir apparaître un domaine pseudo-élastique volumique. La projection des résultats expérimentaux, dans le plan $\left(\mathrm{q} / \mathrm{p}^{\prime}, \mathrm{d \varepsilon} \varepsilon_{\mathrm{y}} / \mathrm{d} \overline{)}\right)$, permet de suivre précisément la loi d'écoulement expérimentale au regard des lois de Roscoe et de Rowe.

Mots-clés : pseudo-élasticité volumique, degré de surconsolidation, dilatance, écoulement plastique, essai triaxial, modèle cam-clay, kaolinite P300.

\section{Strain mechanisms in overconsolidated clay}

The strain mechanisms of an overconsolidated clay (P300) kaolinite) along axisymetric triaxial paths are experimentally characterized with particular interest given to the deviatoric mechanism due to the deviatoric part of stress tensor. The presentation of tests results along pure deviatoric stress paths in a (e, p', q) space and the use of the Cam-clay model allow to follow the evolution of the experimental path and to highlight a pseudo-elastic volumetric domain. The projection of the experimental results in the $\left(\mathrm{q} / \mathrm{p}^{\prime}, \mathrm{d} \varepsilon, / \mathrm{d} \varepsilon\right)$ plane allows a detailed experimental characterization of the plastic flow in comparison with Roscoe and Rowe's relationships.

Key words : volumetric pseudo-elastícity, over consolidation ratio, dilatancy, plastic flow, triaxial test, cam-clay model, kaolinite $\mathrm{P} 300$. 


\section{Introduction}

Les développements récents ont permis la réalisation d'appareils triaxiaux avec systèmes d'asservissement de plus en plus performants rendant possible l'exploration du comportement des sols avec un degré élevé de précision. Le pilotage automatique des essais peut se faire suivant un large éventail de chemins de contraintes ( $\mathrm{p}^{\prime}=$ constante, $\sigma_{3}^{\prime}=$ constante, $\mathrm{e}=$ constante, etc.), et l'acquisition en temps réel des données permet une analyse très fine du comportement aussi bien en petites qu'en grandes déformations. L'objectif du travail présenté dans cet article est de contribuer, par l'observation et en se plaçant dans le cadre formel de l'élastoplasticité, à l'amélioration de la compréhension des mécanismes de déformation dans les argiles surconsolidées.

Sur chemins triaxiaux classiques $\left(\sigma_{3}^{\prime}=\right.$ constante et $e=$ constante) la déformation volumique totale est le résultat d'un couplage entre deux mécanismes, un mécanisme isotrope provoqué par la composante isotrope de la contrainte ( $p^{\prime}$ ) et un mécanisme déviatoire provoqué par la composante déviatoire $(q)$. Une attention particulière est portée au mécanisme déviatoire et à l'apparition et à l'évolution des déformations volumiques dues la partie déviatoire de la contrainte. Les chemins purement déviatoires (menés au triaxial à $\mathrm{p}^{\prime}=$ constante) présentent l'intérêt de permettre de s'affranchir de la partie isotrope de la contrainte et la déformation volumique obtenue est ainsi directement reliée au déviateur.

Les résultats montrent que, quel que soit le chemin. une phase que l'on peut qualifier de pseudo-élastique volumique apparaît en début du chargement. Celle-ci, inexistante dans les argiles normalement consolidées $(\mathrm{OCR}=1)$, correspond à un domaine dans lequel la déformation volumique n'est fonction que de l'évolution de la partie isotrope de la contrainte. Pour une contrainte de consolidation donnée, ceci se traduit, dans l'espace du triaxial (e, p', q), par une surface incurvée issue de la courbe de déchargement isotrope $c_{\text {. }}$ Bien qu'associée, dans certains modèles comme le modèle Cam-clay par exemple, à un comportement élastique non linéaire, elle ne traduit pas la réversibilité du comportement. Nous distinquerons donc le domaine pseudo-élastique volumique, défini ci-dessus et caractérisé en grandes déformations, du domaine élastique caractérisé par la réversibilité du comportement, et mis en évidence dans le domaine des petites déformations, de I'ordre de $10^{-6}$ à $10^{-5}$ de la déformation axiale (Biarez, 1962; Seed et Idriss, 1970; Kokusho, 1980; Hicher, 1985; Vucetic, 1994; Hicher, 1996). Le modèle Cam-clay initial a été introduit comme repère théorique simple permettant une meilleure interprétation des résultats obtenus.

\section{Procédure experimentale et materiau utilisé}

Les essais ont été réalisés sur la kaolinite P300 qui a fait l'objet d'études approfondies permettant notamment de perfectionner l'écriture des lois de comportement (modèle multimécanismes de l'ECP; Hujeux, 1985). Le tableau I résume les paramètres d'identification du matériau.
TABLEAUI Paramètres de nature.

\begin{tabular}{|c|c|}
\hline Composition minéralogique & Kaolinité - Illite - Quartz \\
\hline Couleur & Jaune \\
$\%<80 \mu \mathrm{m}$ & 96 \\
$\%<2 \mu \mathrm{m}$ & 59 \\
Limite de liquidité $(\%)$ & $\mathrm{w}_{\mathrm{t}}=40$ \\
Indice de plasticité $(\%)$ & $\mathrm{Ip}=20$ \\
\hline
\end{tabular}

L'argile, qui au départ se présente sous forme de poudre sèche, est humidifiée afin d'obtenir une boue dont la teneur en eau est égale à 1,5 w . La pâte est ensuite introduite dans un consolidomètre (à double drainage) et consolidée sous une contrainte verticale de $120 \mathrm{kPa}$. L'échantillon est alors taillè en éprouvette cylindrique avec un élancement $\mathrm{h} / \mathrm{d}=1(\mathrm{~h}=\mathrm{d}=35 \mathrm{~mm})$, il est ensuite placé, muni d'un système antifrettage permettant le drainage aux deux extrémités, dans la cellule triaxiale. Le système antifrettage est formé de deux plaques lisses circulaires légèrement graissées sur lesquelles est disposée une couche fine de latex. Cette mise en place (élancement égal à 1 plus un système antifrettage) apparaît comme un compromis optimal pour maintenir l'homogénéité des déformations (Hicher, 1985; Lade et Tsai, 1985; Frost et Yang, 2003), celles-ci pouvant aller jusqu'à $8 \%$ de la déformation axiale.

Le système expérimental comprend une cellule triaxiale, type Bishop (Bishop et Wesley, 1975), et trois contrôleurs pression volume (GDS1, GDS2, GDS3) pour le pilotage et l'acquisition des résultats. En figure 1 sont représentées en trait fort les commandes transmises aux deux contrôleurs « GDS1 » et « GDS2 », le premier pour le contrôle de la contrainte radiale $\sigma_{3}$ le second pour le contrôle de la contrainte axiale $\sigma_{1}$ (générée par la chambre de pression située dans la partie inférieure de la cellule). Le troisième contrôleur « GDS 3 » a la double fonction de contrôle de la contre pression et d'acquisition des variations de volume. Un logiciel assure l'automatisation des essais sur un large éventail de chemins de contraintes ( $\mathrm{p}^{\prime}=$ constante, $\sigma_{3}^{\prime}=$ constante, $\mathrm{e}=$ constante, etc., Hattab et Hicher, 2004), ainsi que l'acquisition des résultats en temps réel. Une procédure de contrôle de microfuites, pouvant être dues à la porosité de la membrane, y est intégrée, celleci est décrite dans Hattab et Hicher, 1995. Les essais triaxiaux sont menés sur des échantillons saturés, la première étape consistant à vérifier la qualité de la saturation par le suivi du coefficient $\mathrm{B}\left(\frac{\Delta \mathrm{u}}{\Delta \sigma_{3}}\right)$ de Skempton; l'étape du chargement isotrope est lancé lorsque $\mathrm{B}=100 \%$.

\section{3}

\section{Caractérisation experimentale du domaine pseudo-élastique volumique}

\section{1}

\section{Définition}

Le comportement pseudo-élastique volumique a été observé et mis en évidence sur chemins triaxiaux classiques (à e et $\sigma_{3}^{\prime}$ constants) dans les argiles surconsolidées par plusieurs auteurs (Ladd, 1964 ; Lee et Seed, 1967 ; Parry et Nodarajah, 1973 ; Biarez et Hicher, 1994). 


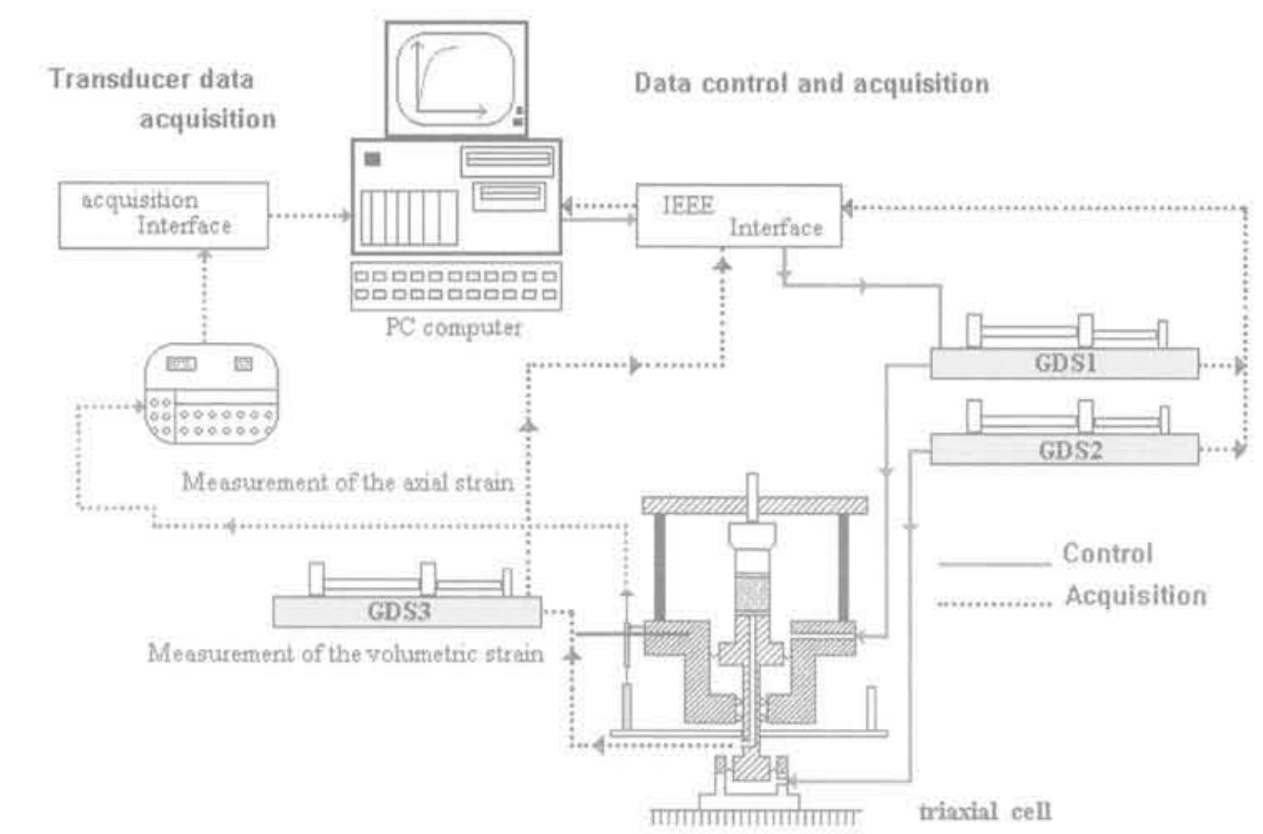

FIG.1 Système expérimental.

Experimental system.

Cette première phase du comportement correspond, lorsque le chemin est à contrainte latérale effective constante $\left(\sigma_{3}^{\prime}=\right.$ constante), à un chemin qui dans le plan $\left(e, p^{\prime}\right)$ suit approximativement la courbe $c_{\text {ede décharge- }}$ ment isotrope (exemple de la kaolinite P300 (Fig. 2)).

Par conséquent, la caractérisation du comportement pseudo élastique volumique observé, sera basée sur l'expression de la courbe de déchargement isotrope $c_{c}$. Ainsi, tout chemin évoluant dans l'espace (e, p', q) et dont la projection sur $\left(e, p^{\prime}\right)$ suit le chemin $c_{s^{\prime}}$ est supposé induire des déformations volumiques dues uniquement à l'évolution de la partie isotrope de la contrainte sans que celles ci soient élastiques, on les notera $d \varepsilon^{(p)}$ (la décomposition du tenseur des contraintes étant donnée par l'équation 1-A en annexe A).

La relation (8-A) de l'annexe A s'exprimera alors sous la forme suivante:

$$
\mathrm{d} \varepsilon_{\mathrm{v}}^{(\mathrm{p})}=-\frac{\mathrm{c}_{\mathrm{s}}}{2,3\left(1+\mathrm{e}_{0}\right)} \frac{\mathrm{dp}}{\mathrm{p}^{\prime}}
$$

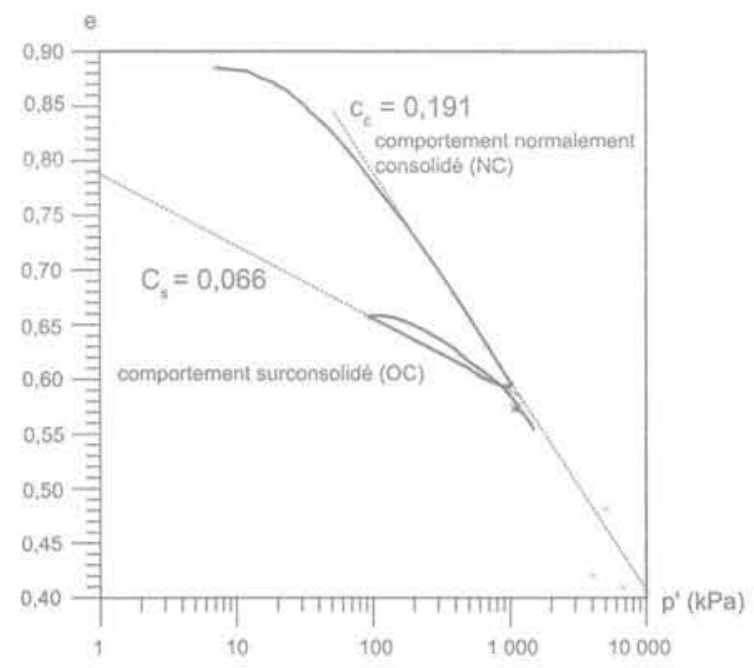

FG. 2 Chemin isotrope chargement-déchargementrechargement sur kaolinite P300.

Isotropic path loading-unloading-reloading on kaolinite P300.
Dans ce qui suit, nous allons voir comment apparaitt le comportement pseudo-élastique dans différents chemins triaxiaux.

\section{2}

\section{Chemins purement déviatoires}

Dans un chemin triaxial axisymétrique purement déviatoire, l'essai consiste à maintenir $p^{\prime}=$ constante. En terme d'incréments, l'équation (1-A) devient $\mathrm{d} \sigma^{\prime}=$ $\mathrm{dq} \mathrm{c}$, par conséquent la déformation volumique obtenue n'est due qu'à l'évolution du déviateur noté de $\varepsilon_{y}^{\text {'q }}$ soit $\mathrm{d} \varepsilon_{\mathrm{v}}=\mathrm{d} \varepsilon_{\mathrm{v}}{ }_{\mathrm{v}}^{(\mathrm{ic})}, \operatorname{car} \mathrm{d} \varepsilon_{\mathrm{v}}{ }^{\left(p^{\prime}\right)}=0$.

Les résultats d'essais à des degrés de surconsolidation variant de 1 à 50 sont représentés dans les plans (q. $\left.\mathrm{p}^{\prime}\right),(\varepsilon, q)$ et (e, log $\left.\mathrm{p}^{\prime}\right)$ (Figs. 3 et 4$)$, la contrainte de consolidation étant $p_{c 0}^{\prime}=1000 \mathrm{kPa}$. Les essais dans les conditions normalement consolidés (NC) à différentes contraintes de consolidation ont permis préalablement de fixer les droites c et $M$ (Hattab, 1995), et les essais surconsolidés $(O C)$ de fixer la droite de plasticité parfaite notée PP dans le plan $\left(e, \log \mathrm{p}^{\prime}\right)$. Dans le modèle de Camclay cette droite correspond à la droite d'état critique CSL. Le parallélisme avec la droite c se trouve ainsi vérifié (Lee et Seed, 1967; Schofield et Wroth, 1968; Biarez et Hicher, 1994; Favre et al., 2002). On déduit les valeurs des paramètres suivants: $M=1,05, c_{c}=0,191$ et $c_{s}=0,066$.

Lorsque les résultats d'essais à degré de surconsolidation supérieur à 1 (noté OCR > 1) sont projetés dans le plan $(\varepsilon, q)$, une première phase est mise en évidence en début du chargement pour laquelle la déformation volumique est nulle (Fig. 3). Ceci se traduit par $\varepsilon_{v}=\varepsilon_{v}^{(i)}=0$ et le tenseur de déformation ne dépend que de la déformation déviatoire $\bar{\varepsilon}$; la relation $(5-\mathrm{A})$ de l'annexe $\mathrm{A}$ devient alors : $\varepsilon=\bar{\varepsilon}$ d. Aux limites de cette phase, l'écoulement plastique apparait par un comportement soit contractant si $\mathrm{OCR}<2,5$, soit dilatant si $\mathrm{OCR}>2,5$. Le $\mathrm{OCR}=1,5$ semble induire un comportement pour lequel $\varepsilon_{\mathrm{y}}=\varepsilon_{\mathrm{v}}(\mathrm{q} \mid=0$ tout le long du chemin, et ce jusqu'à l'état de plasticité parfaite défini par $\mathrm{M}$ dans le plan (q, $\mathrm{p}^{\prime}$ ). 


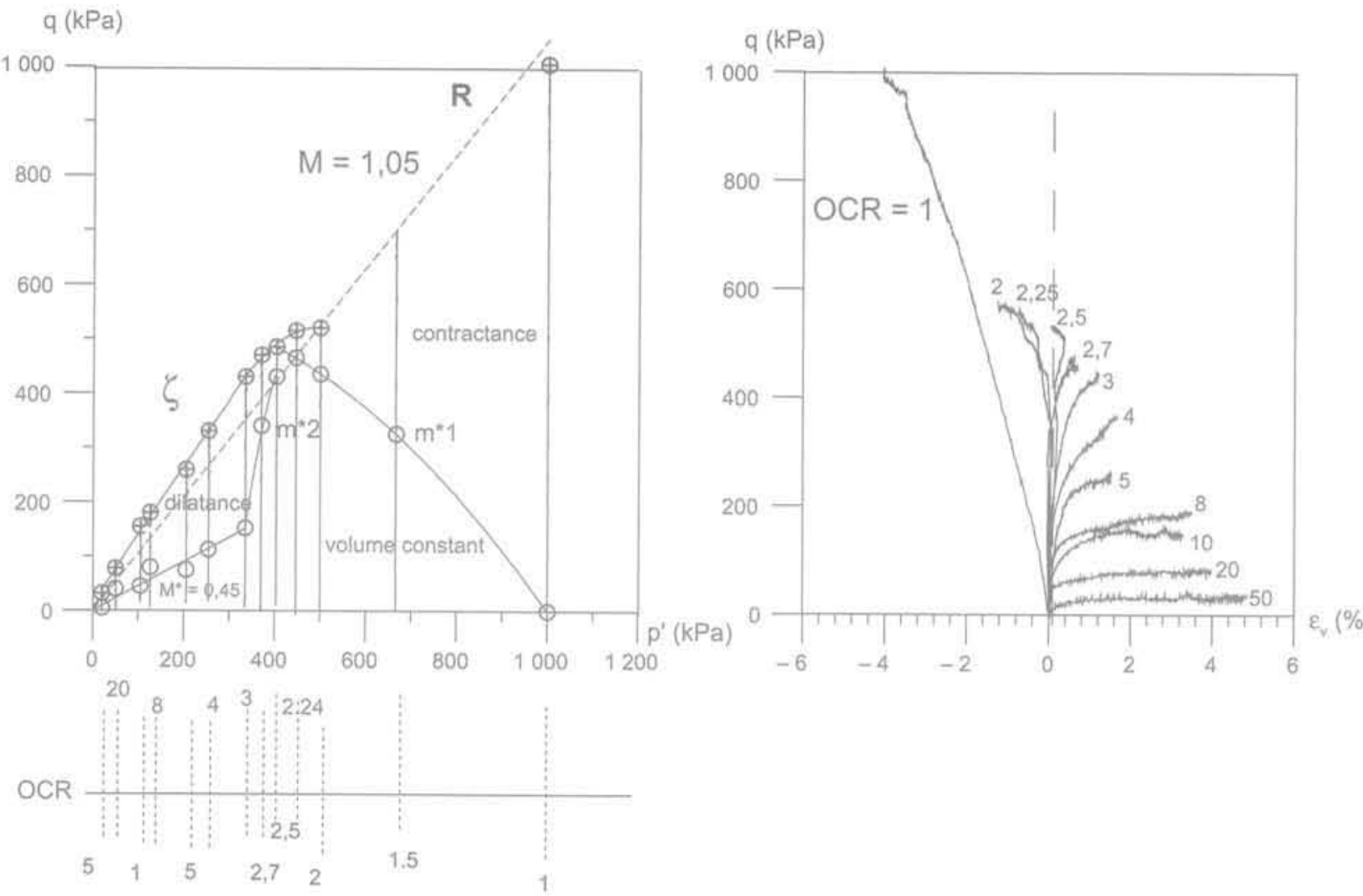

FG. 3 Chemins purement déviatoires à differents OCR. Pure deviatoric paths for different OCR.

Ces résultats, particulièrement l'évolution de la contractance et de la dilatance que l'on peut suivre en figure 3 , ont permis d'identifier trois domaines du plan $\left(q, p^{\prime}\right)$ : un domaine à (cvolume constant ) limité par la courbe expérimentale notée $\left(M^{*} m^{*} 2 m^{*} 1\right)$ dans Hattab et Hicher (2004), où l'évolution du chemin déviatoire n'induit aucune déformation volumique $\left(\varepsilon_{v}=0\right)$. Cette zone est séparée par les domaines de contractance (borné par $\mathrm{m}^{\mathrm{n}} 1$ et la droite d'état critique $\mathrm{M}$ ) et de dilatance (borné gar $\mathrm{M}^{*} \mathrm{~m}^{*} 2$, et la courbe de résistance maximum notée $\zeta$ ). Ce comportement à "volume constant $x$ se traduit, dans le plan (e, p') en figure 4, par

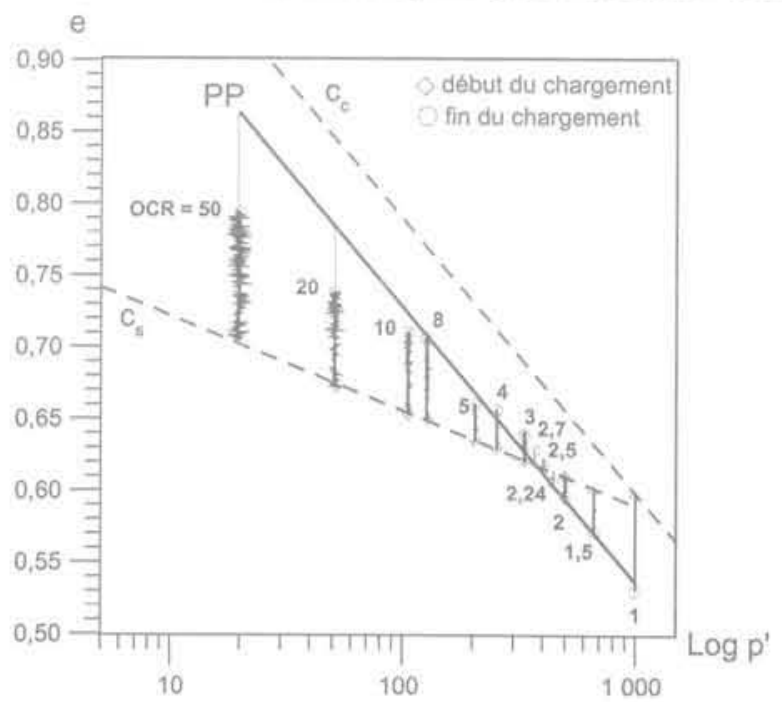

FIG.4 Résultats d'essais à contrainte moyenne effective constante, évolution des chemins dans le plan $\left(\mathrm{e}, \log \mathrm{p}^{\prime}\right)$

Test results on constant mean stress, paths evolutions in $\left(e, \log p^{\prime}\right)$ plane. un point fixe sur la droite $\mathrm{c}_{\mathrm{s}}$. A l'issue de cette première phase, un écoulement plastique est activé dans les conditions de contractance (diminution de e) et de dilatance (augmentation de e) décrites précédemment.

Ainsi ces résultats montrent que sur chemins à $\mathrm{p}^{\prime}=$ constante le comportement pseudo-élastique volumique est un domaine à d $\varepsilon_{\mathrm{v}}=0$ (ou à $\mathrm{e}=$ constante).

\section{3}

\section{Chemins triaxiaux classiques}

Les résultats d'essais triaxiaux à $\sigma_{3}^{\prime}=$ constante sont représentés en figure 5 , pour OCR $=5$ et $\mathrm{OCR}=8$, $\mathrm{p}^{\prime}$. $=1000 \mathrm{kPa}$. Dans les deux cas, nous pouvons suivre dans le plan (e, p'), un chemin évoluant sur la courbe $\mathrm{c}_{\mathrm{s}}$ en début du chargement. Nous retrouvons donc la phase de comportement pseudo-élastique volumique, les déformations volumiques qui s'y développent sont indépendantes du déviateur de contrainte q et vérifient l'équation (1).

Sur chemin non drainé $\left(\varepsilon_{v}=0\right)$, le tenseur de déformation ne dépend que de la déformation déviatoire $\bar{\varepsilon}$, la relation (5-A, annexe $A$ ) devient: $\varepsilon=\tilde{\varepsilon}$ d. Les essais sont réalisés à des degrés de surconsolidation identiques aux essais précédents soit OCR $=5$ et $O C R=8$ (Fig, 6). Dans le plan (q, $\mathrm{p}^{\prime}$ ) les résultats montrent une très faible variation de $p^{\prime}$ en début de chargement, et le chemin suit sensiblement une verticale jusqu'à une certaine valeur du déviateur qu'on nommera $q_{\text {car: }}$. Puis, le chemin s'incurve vers les domaines de p' plus élevés avant de rejoindre l'état de plasticité parfaite (ou état critique). Ces résultats rejoignent ceux de Ladd (1964) sur la «Simple Clay) NC et OC. Dans le plan (e, p'), cette première phase de comportement se traduit par un point fixe sur la courbe $c_{\text {s. }}$ Le comportement pseudo-élastique volumique dans les chemins à est donc caractérisé par un domaine où p' reste constante. 

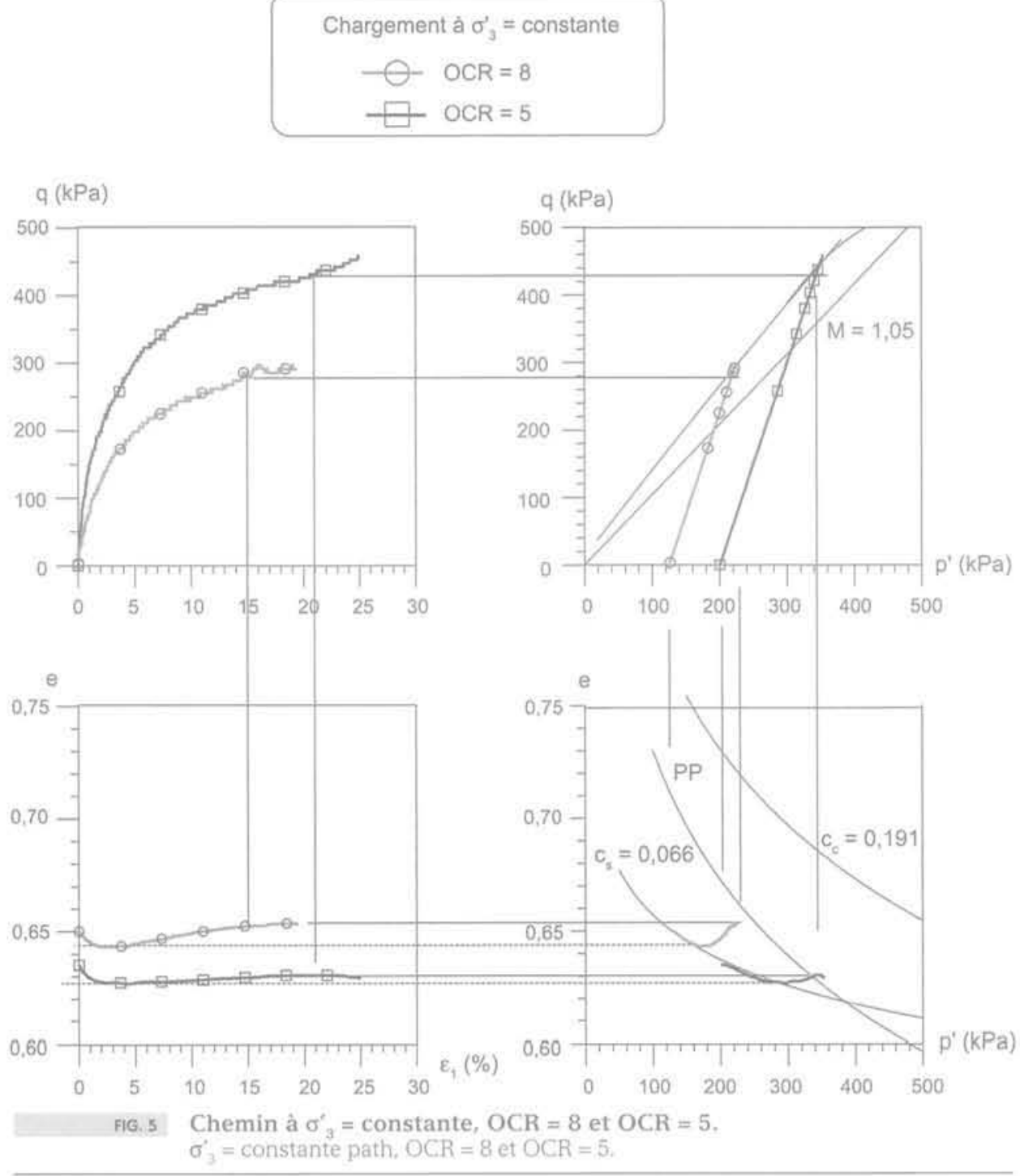

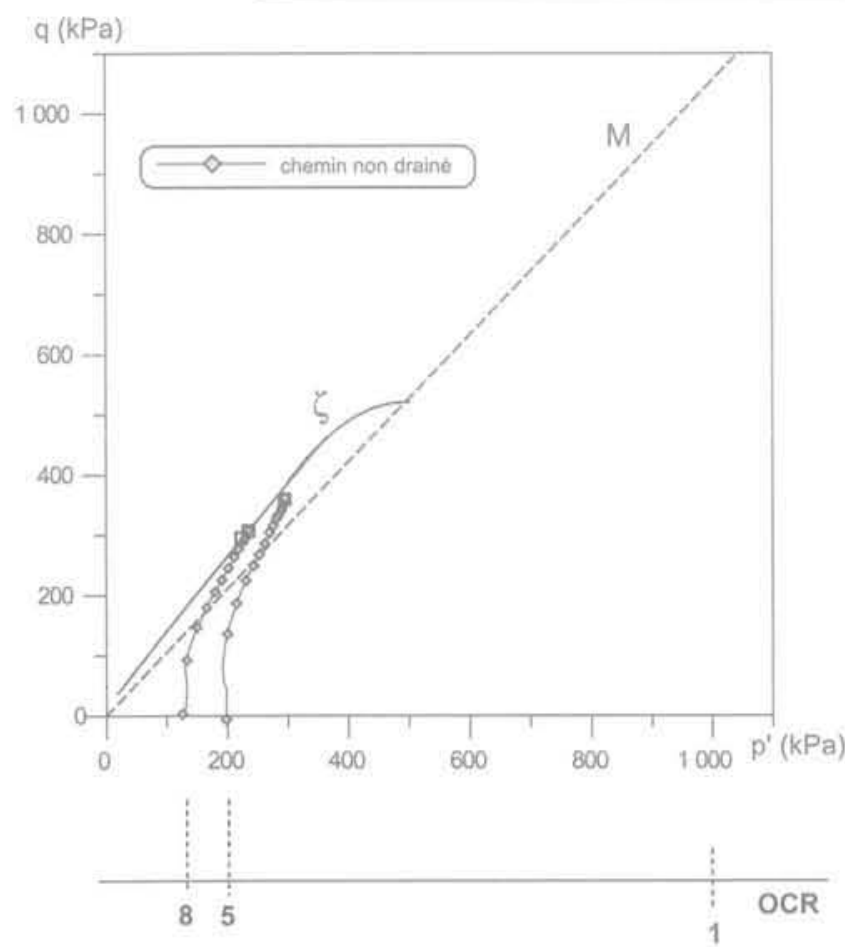

HG.6 Chemin non drainé, $\mathrm{OCR}=8$ et $\mathrm{OCR}=5$. Undrained path, $\mathrm{OCR}=8$ et $\mathrm{OCR}=5$.

\section{Résultats expérimentaux dans l'espace $(e, p$ ', q)}

La représentation des essais triaxiaux, proposée en 3D dans l'espace (e, $p^{\prime}$ ', q), permet une analyse qualitative en référence au modèle Cam-clay, Même si les limites liées au modèle (Aitkinson et Bransby, 1977; Muir-Wood, 2001), qui ressortent ici d'une manière évidente, ont été en partie résolues par un certain nombre de modèles plus sophistiqués à multimécanisme (Hujeux, 1985; Stallebrass et Taylor, 1997, etc.), son utilisation comme repère théorique simple, permet une présentation plus claire de cette étude expérimentale focalisée sur l'existence du domaine pseudo élastique volumique et l'évolution de l'écoulement plastique.

\section{1}

\section{Représentation du domaine pseudo-élastique volumique}

Sur chemin $\mathrm{p}^{\prime}=$ constante, $\mathrm{e}=$ constante ou $\sigma_{3}^{\prime}=$ constante, le comportement pseudo-élastique volumique existe et peut être caractérisé dans le plan 
(e, log $\mathrm{p}^{\prime}$ ) par la courbe $c_{\text {. }}$ Ce sera un point fixe quand les chemins sont à $p^{\prime}=$ constante et $\mathrm{e}=$ constante, et une courbe qui suit $\mathrm{c}_{\mathrm{s}}$ sur un chemin à $\sigma_{3}^{\prime}=$ constante. La figure 7 reprend les résultats pour ces trois chemins, les degrés de surconsolidation étant respectivement $\mathrm{OCR}=8$ et $\mathrm{OCR}=5$. Dans l'espace $\left(e, p^{\prime}, q\right)$ et pour une contrainte de consolidation donnée, le comportement pseudo-élastique volumique se traduit par une surface incurvée notée $C^{3 \mathrm{~d}}$ issue de la courbe de déchargement isotrope $\mathrm{c}_{s^{\prime}}$ sa représentation est donnée en figure 8. Ainsi, sur chemin triaxial classique (à $\sigma_{3}^{\prime}=$ constante), par exemple, et pour un OCR donné, les points représentant la phase pseudo-élastique volumique seront l'intersection entre le plan $\sigma_{3}^{\prime}=$ constante et la surface $\mathrm{C}_{5}^{3 d}$. L'écoulement plastique s'active dès que la droite obtenue atteint sur la surface $\mathrm{C}_{\mathrm{s}}^{3 \mathrm{~d}}$ la limite du domaine pseudo-élastique. D'une manière générale, quel que soit le chemin de contrainte suivi, la phase pseudo-élastique volumique sera définie par l'intersection entre le plan du chemin donné $\left(\sigma_{3}^{\prime}=\right.$ constante, $e=$ constante, $\mathrm{p}^{\prime}=$ constante) et la surface $\mathrm{C}_{\mathrm{s}}^{3 \mathrm{~d}}$.

Le modèle Cam-clay, dont les principes sont rappelés en annexe B, l'associe à un comportement élastique non linéaire défini par la relation (8-A) (annexe A) et limité par la surface de charge de Roscoe (annexe B). La surface pseudo-élastique volumique $\mathrm{C}_{s}^{3 \mathrm{~d}}$ ne traduisant pas la réversibilité du comportement, ne peut être associée à un comportement élastique, il n'est donc pas question ici de discuter de la validité du domaine élastique de Cam-clay. Toutefois, l'intérêt de celui-ci, dans cette étude, se situe dans son écriture basée sur la définition même établie pour la surface $C{ }_{4}^{3 d}$ par la relation (1) (caractérisation expérimentale en grandes déformations traduisant un comportement spécifique sur chemin triaxial qui n'est liée qu'à l'évolution de la partie isotrope de la contrainte).

Les hypothèses et développements du modèle Camclay permettent de construire la surface de charge et la ligne d'état critique en 3D, dans l'espace (e, p', q) et dans un intervalle donné de l'indice des vides e (Fig. 1B, annexe B). Dans ce qui suit, cette surface sera représentée avec les chemins expérimentaux, dans l'espace (e, p', q).

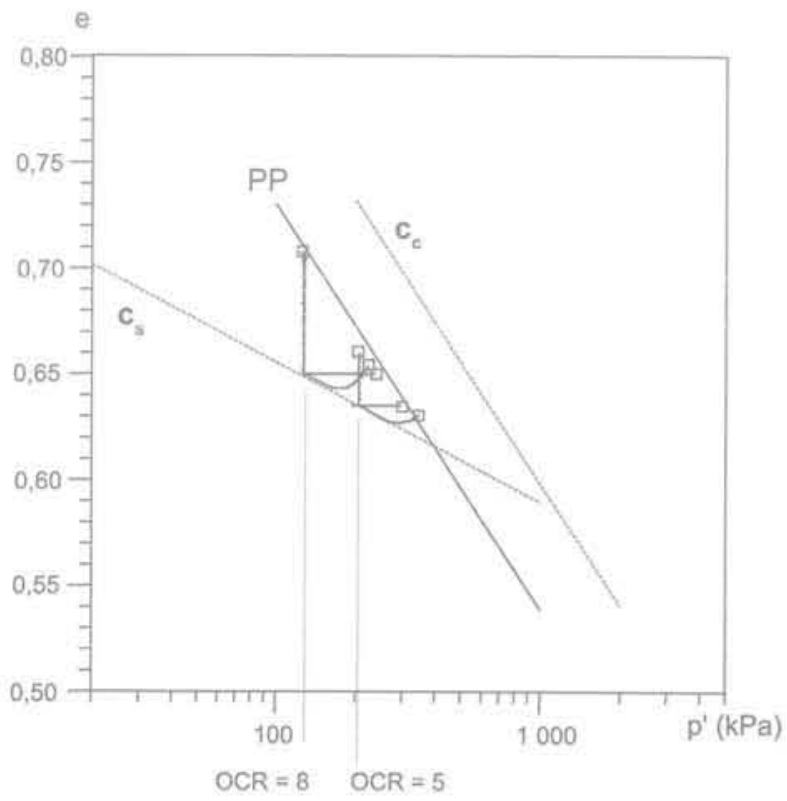

FIG.7 Différents chemins de contrainte dans (e, $\left.\mathrm{p}^{\prime}\right)$. $\mathrm{OCR}=8$ et $\mathrm{OCR}=5$.

Different stress path in $\left(e, p^{\prime}\right), O C R=8$ et $O C R=5$.

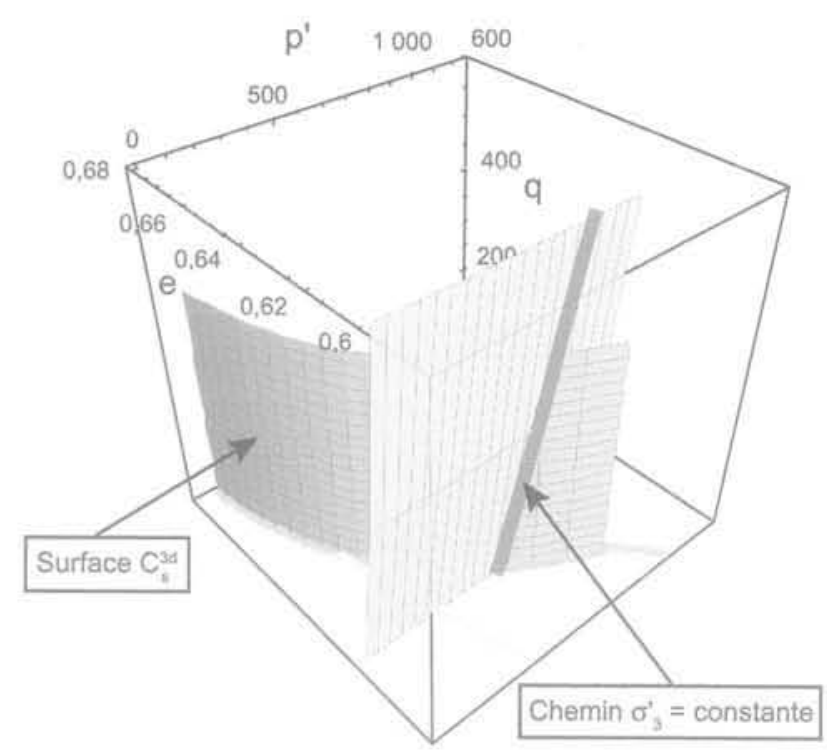

FG.8 Représentation du domaine élastique de Cam-clay (surface $\mathrm{C}_{\mathrm{s}}^{3 \mathrm{~d}}$ ) dans $\left(\mathrm{e}, \mathrm{p}^{\prime}, \mathrm{q}\right)$. Representation of Cam-clay elastic domain (Cs surface) in $\left(e, p^{\prime}, q\right)$.

\section{2}

\section{Résultats d'essais à p' constant}

Les résultats d'essais surconsolidés, sur chemins purement déviatoires, sont représentés dans (e, p', q) par intervalle d'indice des vides et suivant des orientations du repère propre à chaque cas (Figs. 10, 11, 12, $13,14)$. Les positions que prennent les courbes expérimentales, sur la courbe de déchargement isotrope $\mathrm{c}_{\mathrm{s}}$ nous permet de localiser aisément le degré de surconsolidation correspondant. La figure 9 représente dans (e, p', q) l'état critique expérimental (ou la courbe de plasticité parfaite), obtenu à partir des résultats des

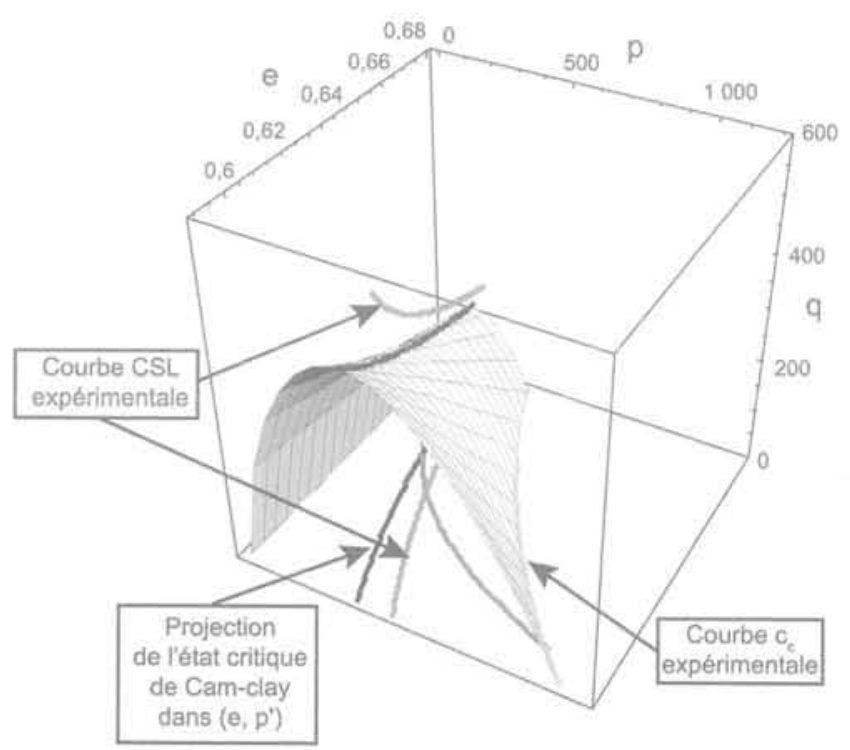

FIG.9 Représentation de la surface de Roscoe dans (e, p', q), et projection de la courbe expérimentale de chargement et déchargement isotrope $\mathrm{p}^{\prime}=1000 \mathrm{kPa}$. Roscoe surface representation in (e, $\left.p^{\prime}, q\right)$, and projection of experimental curves on isotropic loading and unloading, $\mathrm{p}_{\mathrm{co}}^{\prime}=1,000 \mathrm{kPa}$. 


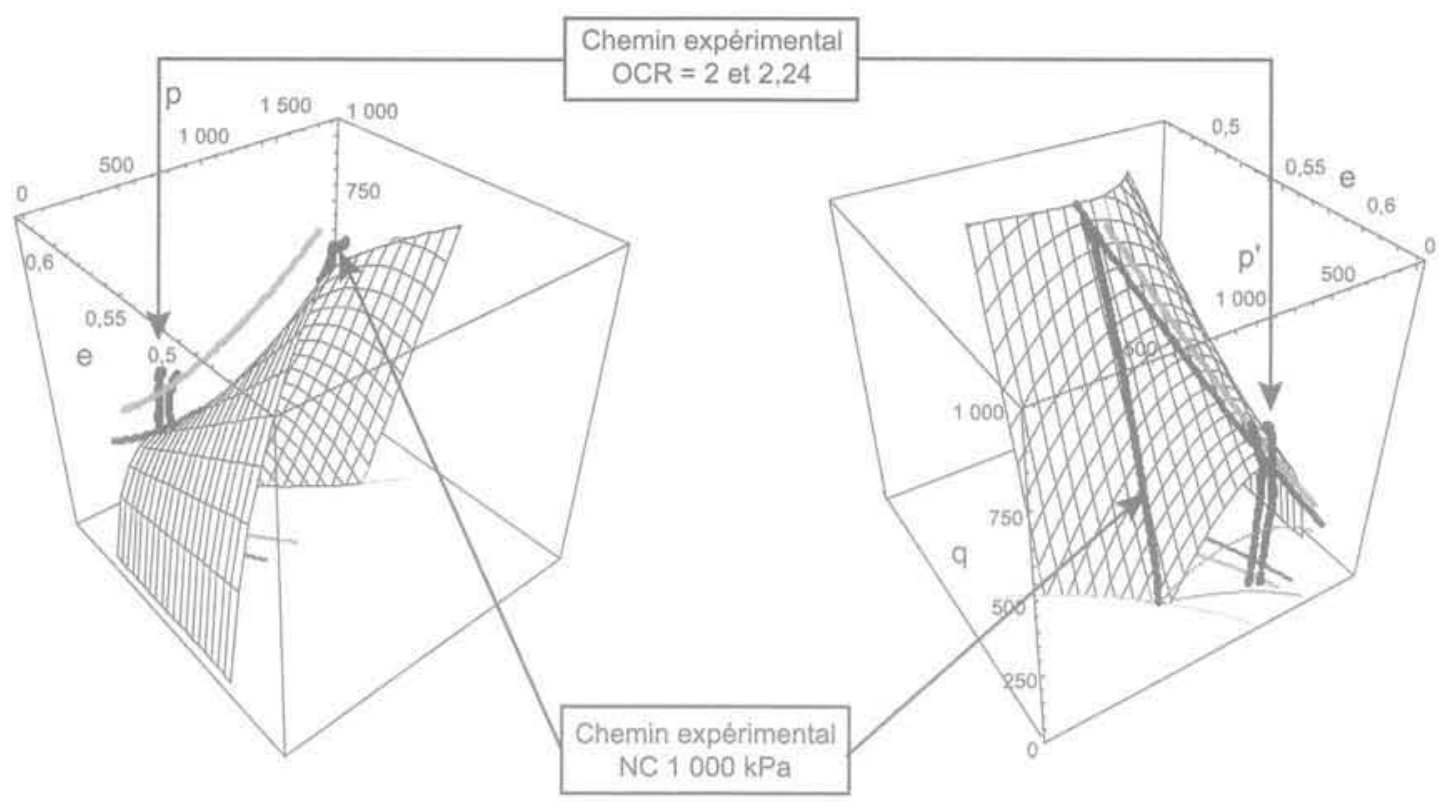

FG. 10 Projection des résultats d'essai expérimentaux ( $\mathrm{p}^{\prime}=$ constante), argile NC1000 et $\mathrm{OCR}=2 ; 2,24$, dans $\left(\mathrm{e}, \mathrm{p}^{\prime}, \mathrm{q}\right)$.

Projection in (e, $\left.p^{\prime} q\right)$ of ( $p^{\prime}=$ constante) test results, $N C 1000$ and $O C R=2,2.24$.

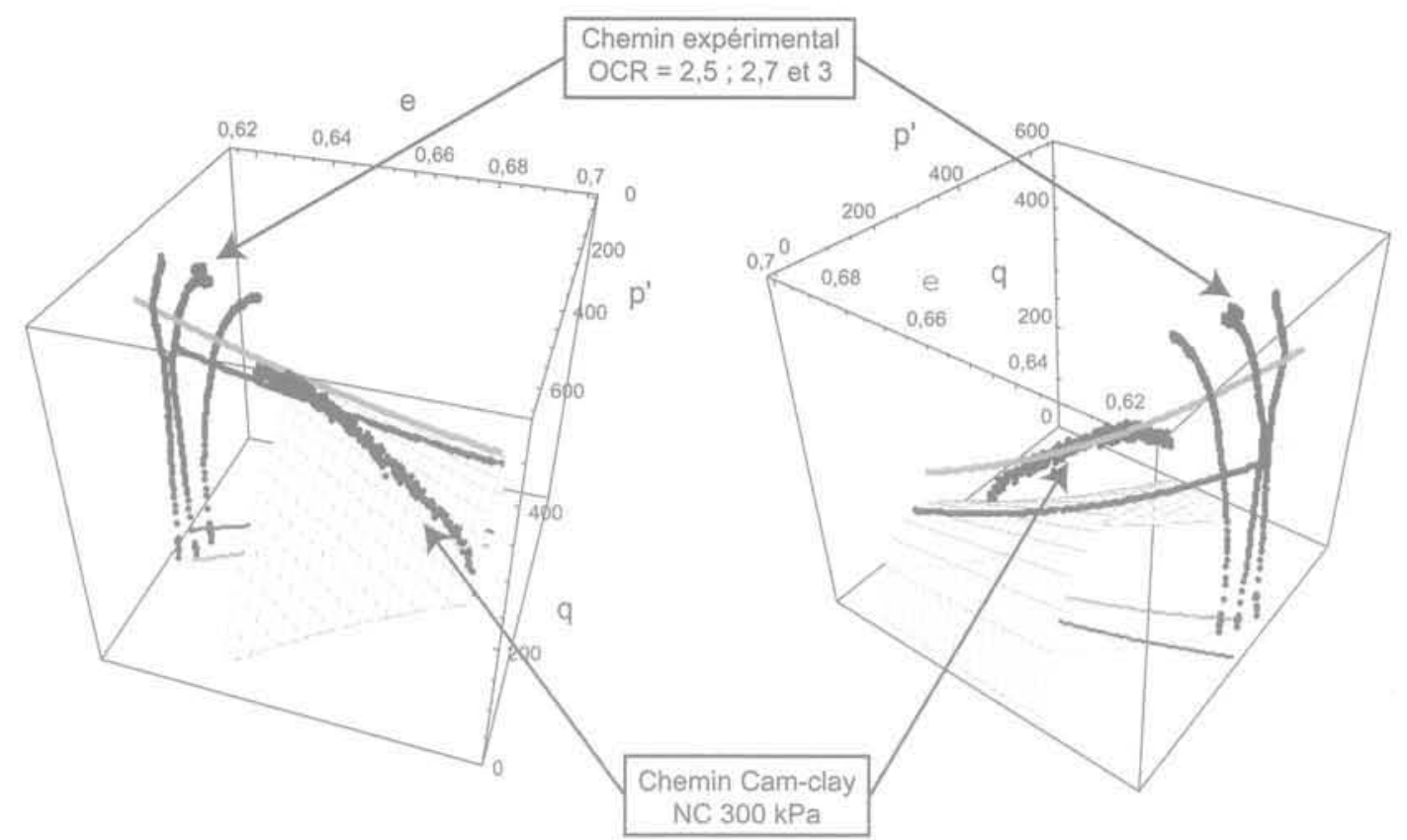

fiG. 11 Projection des résultats expérimentaux ( $\mathrm{p}^{\prime}=$ constante), argile NC 300 et $\mathrm{OCR}=$ 2,$5 ; 2,7 ; 3 ;$ dans $\left(e, p^{\prime}, q\right)$.

Projection in (e, $\left.\mathrm{p}^{\prime} \mathrm{q}\right)$ of $\left(\mathrm{p}^{\prime}=\right.$ = constante test result : $\mathrm{NC} 300$ and $\mathrm{OCR}=2.5,2.7,3$.

figures 3 et 4 . Un décalage évident est observé entre la courbe expérimentale et la courbe d'état critique de Cam-clay (annexe B), L'écart entre la courbe de chargement isotrope et la courbe d'état critique expérimentale dans le plan (e, p'), se trouve ainsi surestimé par le modèle comparé aux résultats expérimentaux (Fig. 4). Toutefois, un calcul par le modèle Cam-clay initial prenant en compte une valeur de OCR égale à 2 (cas du Cam-clay modifié) et non égale à e $(=2,7)$ pour la ligne d'état critique, réajuste sa position par rapport à la courbe expérimentale.

\subsection{1}

\section{Argile normalement consolidée et faiblement surconsolidée}

Les courbes obtenues dans les essais normalement consolidés sous une contrainte de consolidation de $1000 \mathrm{kPa}$ et $300 \mathrm{kPa}$, notées respectivement NC1000 (Fig. 10) et NC300 (Fig. 11), montrent que le chemin expérimental évolue non loin de la surface de Roscoe dont la forme est parfaitement restituée particulièrement en début de chargement. Ceci est très visible sur 
la courbe NC1000 (dont la contrainte de consolidation est égale à $1000 \mathrm{kPa}$ ), les deux courbes NC rejoignent ensuite la ligne d'état critique expérimentale.

Les résultats sur l'argile faiblement surconsolidée, présentés en figure 10 (courbes OCR $=2$ et 2,24 ) et en figure 11 (courbes OCR $=2,5 ; 2,7$ et 3 ), montrent un chemin expérimental, dont une partie importante se trouve à l'intérieur de la surface de Roscoe, évoluant dès le début du chargement dans un domaine pseudoélastique volumique (avec e constant et p' constant). Ensuite, très proche de la ligne d'état critique expérimentale, le chemin s'incurve, mettant en évidence un écoulement légèrement contractant pour les OCR de 2; 2,24 , et dilatant pour les OCR de 2,7 et 3 . La valeur 2,5 apparaît comme une valeur singulière du degré de surconsolidation OCR, pour laquelle la phase pseudo-élastique volumique ( $\mathrm{p}$ ' constante e constant) se poursuit jusqu'à la rupture. Comme schéma de référence, la figure 1-B de l'annexe $B$ représente les domaines de contractance et de dilatance sur la surface de Roscoe.

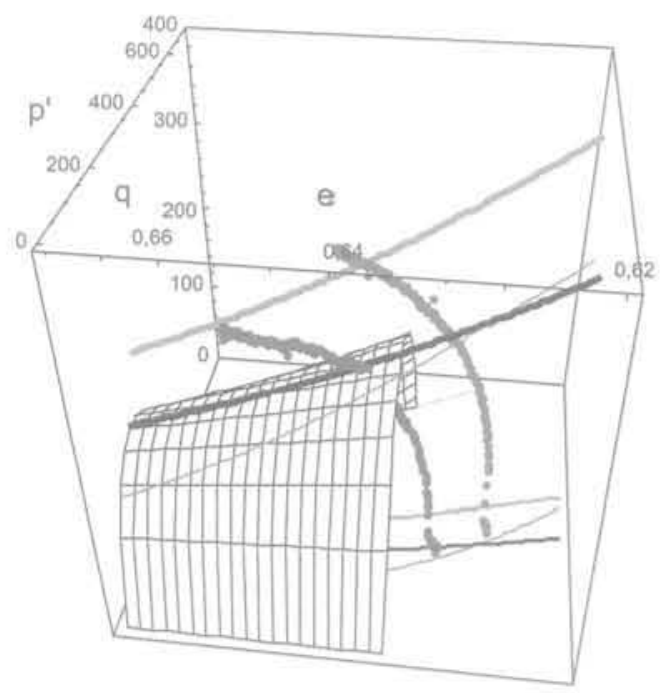

Fig. 12 Projection des résultats expérimentaux $\left(\mathrm{p}^{\prime}=\right.$ constante) argile $\mathrm{SC}$ et $\mathrm{OCR}=4,5$. Projection of $\left(\mathrm{p}^{\prime}=\right.$ constante) test results $\mathrm{OC}$ clay, $\mathrm{OCR}=4,5$.

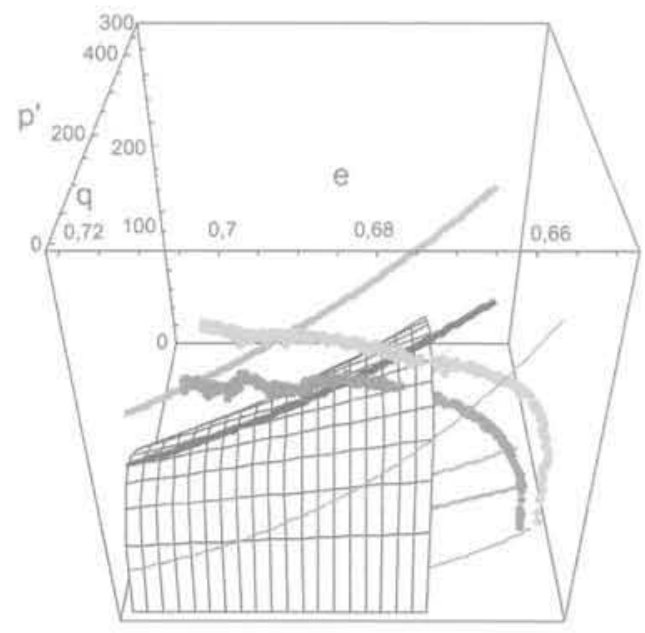

FIG. 13 Projection des résultats expérimentaux $\left(\mathrm{p}^{\prime}=\right.$ constante) argile $\mathrm{SC}$ et $\mathrm{OCR}=8,10$. Projection of ( $p^{\prime}=$ constante) test results $O C$ clay $\mathrm{OCR}=8,10$.

\section{Alan:}

\section{Argile fortement surconsolidée}

Les figures 12, 13 et 14 présentent les résultats d'essai correspondant à des valeurs élevées de OCR = 4, 5 (Fig. 12), 8, 10 (Fig. 13) et 20, 50 (Fig. 14). Pour toutes ces valeurs du OCR, la phase à ' $c p^{\prime}$ constant e constant » de la courbe expérimentale observée en début de chargement met en évidence un domaine pseudo-élastique volumique comparable (mais plus réduit) à la surface de charge proposée par Roscoe. L'écoulement en dilatance s'active ensuite en accord avec le concept d'état caractéristique de Luong (1978) et Ishihara et Okada (1978). Tous les chemins expérimentaux semblent converger vers la ligne d'état critique (expérimentale ou calculée en prenant $\mathrm{OCR}=2$ ) en fin de chargement.

\section{A. 0.8}

\section{Écoulement plastique}

L'écoulement plastique, sur chemin à p' = constante pour les OCR variant de 1 à 50 , est représenté dans le plan ( $\mathrm{q} / \mathrm{p}^{\prime}, \mathrm{d} \varepsilon_{\mathrm{v}}^{\mathrm{p}} / \mathrm{d} \bar{\varepsilon}^{p}$ ) (Fig, 15), Les incréments de déformations n'étant dus qu'à l'évolution de q, nous considérons qu'ils sont essentiellement de type plastique.

Nous représentons également dans ce repère ( $q / p^{\prime}$, $\left.\mathrm{d} \varepsilon_{\mathrm{v}}^{p} / \mathrm{d} \bar{\varepsilon}^{\mathrm{p}}\right)$ la loi d'écoulement du modèle Cam-clay, celleci est une droite passant par $\left(\mathrm{q} / \mathrm{p}^{\prime}=\mathrm{M}\right)$ vérifiant l'équation (4-B) du tableau II.

Lorsque la loi d'écoulement selon Rowe est traduite dans ce même plan (relation (2-C) de l'annexe C), on obtient une courbe qui rejoint progressivement Camclay en grandes déformations, les deux courbes se confondent alors entre l'état $q / p^{\prime}=M$, et la résistance maximale $\mathrm{q}_{\max } / \mathrm{p}^{\prime}$ dans le domaine dilatant.

La figure 15 montre un comportement très différent selon que le degré de surconsolidation est fort, faible ou égal à 1. Pour l'argile normalement consolidée, l'écoulement est linéaire avec une droite de pente

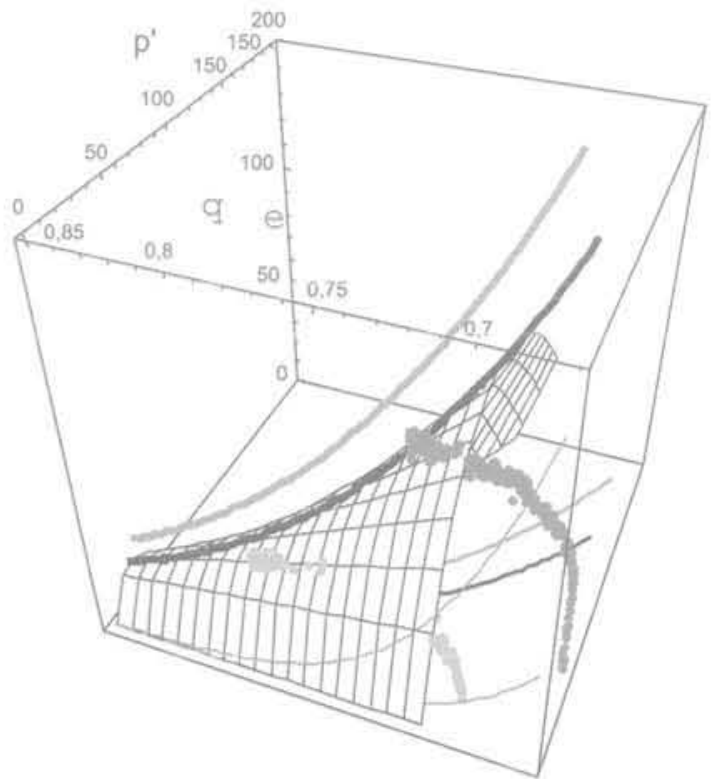

FlG. 14 Projection des résultats expérimentaux ( $\mathrm{p}^{\prime}=$ constante) argile $\mathrm{SC}$ et $\mathrm{OCR}=20,50$. Projection of $\left(\mathrm{p}^{\prime}\right.$ ' = constante) test results OC clay OCR $=20,50$. 


$$
\begin{array}{ll}
\hline \ldots & \text { Cam-clay } \\
\ldots \ldots & \text { Argile NC } \\
\ldots \ldots \ldots & \text { Rowe }
\end{array}
$$

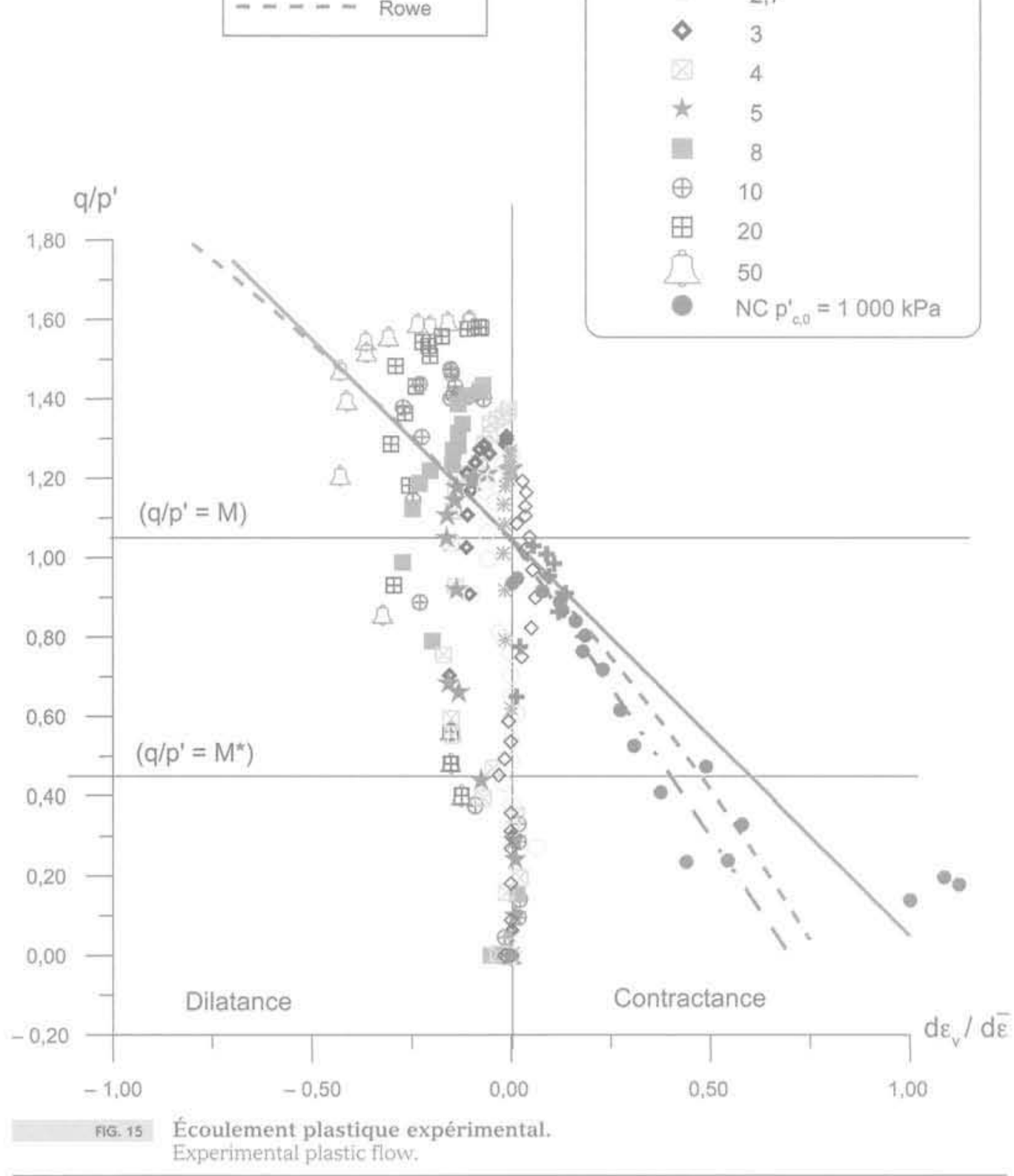

$\alpha=1,5$ au lieu de 1 pour le modèle Cam-clay. L'écoulement selon Rowe est nettement plus proche de la droite expérimentale. L'argîle faiblement surconsolidée $(\mathrm{OCR}=2 ; 2,24)$ présente un écoulement similaire à l'argile NC, mais aussi aux deux lois Cam-clay et Rowe.

Pour ce qui concerne les argiles très surconsolidées $(\mathrm{OCR}=50 ; 20 ; 10 ; 8 ; 5 ; 4 ; 3)$, l'écoulement plastique semble d'abord évoluer linéairement dès que $q / p^{\prime}=M^{*}$ (cet état délimite le domaine pseudo-élastique volumique pour les OCR élevés, Fig. 3). La variation prend ensuite une autre tendance pour venir progressivement rejoindre une valeur $q / p^{\prime}$ maximale (maximum de résistance). A partir de cet état l'efficacité du système d'anti-frettage peut être mis en doute; on se trouve en effet avec des déformations supérieures à $10 \%$ de la déformation axiale et l'interprétation devient difficile. Pour la plupart des cas, principalement pour les valeurs les plus élevés du OCR, le premier point traduisant $\mathrm{q}_{\max } / \mathrm{p}^{\prime}$ se trouve sur la droite d'écoulement de Cam-clay (ou sur la courbe de Rowe, les deux modèles étant dans cette partie du chargement très proches). En se basant sur l'écoulement observé dans le cas des argiles faiblement surconsolidées, l'hypothèse selon laquelle une fois la droite Cam-clay atteinte, les points redescendent en suivant cette droite vers l'état de plasticité parfaite pourrait convenir.

\section{5}

\section{Conclusion}

La représentation des résultats expérimentaux dans l'espace $\left(e, p^{\prime}, q\right)$ offre l'avantage de donner une meilleure visibilité des comportements observés au regard d'un modèle repère simple, celui de Cam-clay. 
Les résultats d'essais dans le cas du chargement à p' constant, permettent, par une analyse qualitative du comportement, une meilleure compréhension du phénomène de contractance et de dilatance. La présentation des résultats expérimentaux avec le modèle élastoplastique Camclay renforce cette analyse, même si celle-ci fait ressortir par ailleurs les limites du modèle, en particulier pour les fortes valeurs du degré de surconsolidation OCR.

Les essais triaxiaux purement déviatoires permettent de distinguer, dans l'espace (e, p', q), les deux domaines de comportement suivants:

a) le domaine pseudo-élastique volumique, mis en évidence par la surface incurvée $C_{s}^{3 d}$, et qui est caractérisé par une évolution du chemin à déformation volumique nulle. La limite supérieure de ce domaine mis en évidence expérimentalement est comparable (mais plus faible) à la surface proposée par Roscoe;

b) l'écoulement plastique, dont l'évolution est mise en évidence dans l'espace (e, p', q), se caractérise par une contractance pour les faibles valeurs de OCR et une dilatance pour les fortes valeurs. Comme tous les chemins expérimentaux semblent converger vers l'état de plasticité parfaite (état critique), les limites de ce domaine d'écoulement peuvent être fixées par la ligne de plasticité parfaite, d'une part, et la limite supérieure du domaine pseudo-élastique volumique, d'autre part.

L'analyse de l'écoulement plastique expérimental dans le plan $\left(\frac{q}{p^{\prime}}, \quad \frac{d \varepsilon_{v}^{p}}{d \bar{\varepsilon}^{p}}\right)$ montre un passage progressif entre le domaine pseudo-élastique volumique, défini par $\frac{d \varepsilon_{v}^{p}}{d \bar{\varepsilon}^{p}}=0$ et le maximum de résistance $\frac{g_{\max }}{p^{\prime}}$. A partir de ce point, l'écoulement pourrait devenir linéaire et conforme à l'écoulement selon les modèles de Cam-clay ou de Rowe, pour atteindre l'état critique $\frac{\mathrm{q}}{\mathrm{p}}=\mathrm{M}$.

\section{Bibliographie}

Aitkinson J.H., Bransby P.L. - The Mechanics of soils. Mac Graw Hill, Cambridge Un. Press, 1977.

Biarez J. - Contribution à l'étude des propriétés mécaniques des sols et des matériaux pulvérulents. Thèse d'État, Grenoble, 1962.

Biarez J., Hicher P.Y. - Elementary mechanics of soils behavior. Saturated remoulded soils. A.A. Balkema, Rotterdam, Brookfield, 1994

Bishop A.W., Wesley L.P. - A hydraulic triaxial apparatus for controlled stress path testing. Géotechnique, 25 (4), 1975. p. $657-670$.

Favre, J.-L., Biarez J., Mekkaoui S.-Models for large strain behaviour of sands and remoulded clays in oedometer and triaxial tests. PARAM 2002 International Symposium. Presses des Ponts et Chaussées/LCPC, 2002, p. 369-385.

Frost J.D., Yang C.T. - Effect of end platens on microstructure evolution in dilatant specimens. Soils and Foundations, $n^{\circ} 43$ (4), 2003, p. 1-11.

Hattab M. - Etude expérimentale du comportement dilatant des argiles surconsolidées. Thèse de doctorat, Ecole centrale Paris Châtenay-Malabry, 1995.

Hattab M., Hicher P.-Y. - Experimental study of the dilatancy in overconsolidated clay. Proceedings of the internafional symposium on compression and consolidation of clayey soils, Hiroshima'95, Japan, A.A. Balkema. Rotterdam, Brookfield, 1995, p. 57-62.
Hattab M. Hicher P.-Y. - Dilating behavjour of overconsolidated clay. Soils and Foundations vol. 44, $n^{\circ} 4,2004, p .27-40$.

Hicher P.-Y. - Comportement mécanique des argiles saturées sur divers chemins de sollicitations monotones et cycliques application à une modélisation élastoplastique et viscoplastique. Thèse de doctorat d'Etat ès sciences physiques. Paris 6, 1985

Hicher P.-Y. - Elastic properties of soils. Journal of Geotechnical Engineering, vol. $122, n^{\circ} 8,1996$, p. 641-648.

Hujeux J.-C. - Une loi de comportement pour le chargement cyclique des sols. Génie parasismique, ss la dir. de V. Davidovici, Presses ENPC. 1985 D. 278-302

Ishihara K., Okada S. - Effects of stress history on cyclic behavior of sands. Soils and Foundations, 18, 4, 1978, p. 31-45.

Kokusho T. - Cvclic triaxial test of dynamic properties of soft clays for wide strain range. Soils and Foundations, vol. 20. $n^{\circ} 2,1980$, p. 45-60.

Ladd C. C. - Stress-Strain behaviour of saturated clay and basic strength principle. Rec. Rapp. R64-17, n² 1. Part 1. MIT, 1964.

Lade P.V., Tsai J. - Effects of localization in triaxial tests on clays. Proc. ICSMFE. San Franscisco, vol. 1, 1985, p. 549-552. Lee K.H., Seed H.B. - Undrained strength characterictics of cohesionless soils. Proc. ASCE, vol. 93, n SM6, 1967, p. 333-360. Luong M.P. - État caractéristique du sol. CR Ac. Sc., t. 287 série B, 1978, p. 305-307.
Muir-Wood D. - Geotechnical Modelling (Applied geotechnics). Ed. Hardcover, 2001.

Parry, N. - Observations on laboratory prepared, overconsolidated specimens of kaolin. Geotechnique, 24, 3, 1973, p. 345358.

Roscoe K.H., Schofield A.N., Wroth C.P. 1958. $\alpha$ On the Ylelding of soils $)$. Geotechnique, vol. $8, n^{\circ} 1,1958, p .22-53$.

Rowe P.W. - The stress dilatancy relation for static equilibrium of an assembly of particles in contact. Proc. of the Royal Society, A269, 1962 , p. 500-527.

Seed H.B., Idriss I.M. - Soil moduli and damping factors for dynamic response analyses. Report EERC-70-10, Earthquake Engineering Research Center. University of California. Berkelev, CA. 1970.

Schofield A.N., Wroth C.P. - Critical state soil mechanics. Mc Graw Hill. New York, 1968

Stallebrass S.E., Taylor R.N. - The development and evaluation of a constitutive model for the prediction of ground movements in overconsolidated clay. Geotechnique, vol. 47, n², 1997, p, 235-253.

Vucetic M. - Cyclic threshold shear strains in soils, Journal of Geotechnical Engineering ASCE, vol. 120, $n^{\circ} 12,1994, p .2208-2228$.

Zienkiewicz O.C.. Naylor D.J. - The adaptation of critical state soil mechanics theory for use in finite elements. Stressstrain behaviour of soils. R.H.G. Parry ed. G.T. Foulis and Co. Henley-onThames England, 1972, p. 537-547. 


\section{Annexes}

\section{A}

\section{Bases théoriques}

\section{A.t}

\section{Description des états de contraintes et de déformations}

Dans un chargement triaxial (axisymétrique de révolution), les contraintes et les déformations sont principales et les tenseurs $\sigma^{\prime}$ et $\varepsilon$ qui représentent respectivement le tenseur de contraintes effectives et le tenseur de déformations, sont diagonales. Par ailleurs, le tenseur des contraintes peut se décomposer en une partie isotrope et une partie déviatoire comme suit:

$\begin{aligned} & \sigma^{\prime}=\mathrm{p}^{\prime} \mathrm{I}+\mathrm{q} \underline{\underline{\mathrm{c}}} \\ & \text { Iest la matrice identité, } \mathrm{c}=\left(\begin{array}{c}\frac{2}{3} \\ -\frac{1}{3} \\ -\frac{1}{3}\end{array}\right) \quad \mathrm{q}=\sigma_{1}^{\prime}-\sigma_{3}^{\prime} \text { et } \mathrm{p}^{\prime}=\frac{\sigma_{1}^{\prime}+2 \sigma_{3}^{\prime}}{3}\end{aligned}$

L'état de contrainte peut donc être décrit par les deux variables p' et q, respectivement contrainte moyenne effective et déviateur des contraintes.

De même, le tenseur de déformation peut se décomposer en une partie isotrope et une partie déviatoire :

$$
\begin{aligned}
& \underline{\underline{\varepsilon}}=\frac{\varepsilon_{\mathrm{v}}}{3} \underline{\underline{I}}+\underline{\underline{\underline{\varepsilon}}} \\
& \varepsilon_{\mathrm{v}}=\varepsilon_{\mathrm{j}}+2 \varepsilon_{3} \\
& \underline{\underline{\underline{\varepsilon}}}=\underline{\underline{\varepsilon}}-\frac{\varepsilon_{\mathrm{v}}}{3} \underline{I}
\end{aligned}
$$

$\varepsilon_{\mathrm{v}}$ étant la déformation volumique totale calculée comme la trace de $\varepsilon$ et $\bar{\varepsilon}$ la partie déviatoire du tenseur de déformation.

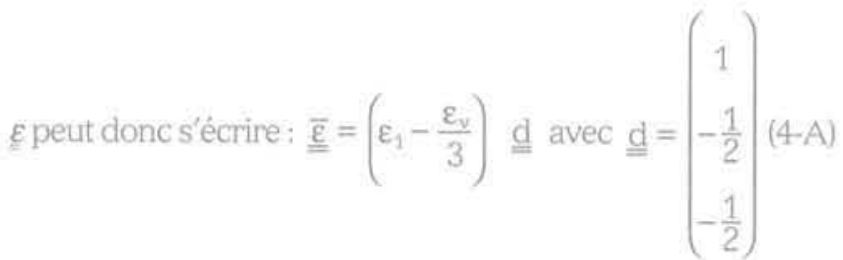

(4-A) montre que le tenseur déviatoire de la déformation est défini par la seule variable scalaire $\bar{\varepsilon}=\varepsilon_{1}-\frac{\varepsilon_{y}}{3}$. L'état de déformation peut ainsi être défini par sa composante isotrope $\varepsilon_{\mathrm{v}}$ et sa composante déviatoire $\bar{\varepsilon}$, comme suit:

$$
\underline{\underline{\varepsilon}}=\frac{1}{3} \varepsilon_{\mathrm{v}} \underline{\mathrm{I}}+\bar{\varepsilon} \underline{\underline{\mathrm{d}}}
$$

\section{An?}

\section{Chemin isotrope}

Les contraintes sur chemin isotrope, sont telles que $\sigma_{1}^{\prime}=\sigma_{2}^{\prime}=\sigma_{3}^{\prime}$ (soit $q=0$ ), et les déformations supposées telles que $\varepsilon_{1}=\varepsilon_{2}=\varepsilon_{3}(\tilde{\varepsilon}=0)$, les relations (1-A) et (5-A) deviennent alors :

$$
\underline{\underline{\sigma^{\prime}}}=p^{\prime} \underline{\underline{I}} \quad \text { et } \underline{\underline{\varepsilon}}=\frac{1}{3} \varepsilon_{\mathrm{v}} \underline{\underline{I}}
$$

Par conséquent, $\varepsilon_{y}$ est directement liée à p', et la courbe obtenue en chargement-déchargement (Fig. 2), se définie par le système d'équation suivant :

$$
\left\{\begin{array}{l}
e=e_{\lambda}-c_{c} \log \left(\frac{p^{\prime}}{p_{1}^{\prime}}\right) \\
e=e_{x}-c_{s} \log \left(\frac{p^{\prime}}{p_{1}^{\prime}}\right)
\end{array}\right.
$$

$c_{c}$ et $c_{s}$ sont respectivement l'indice de compression et l'indice de gonflement, $p^{\prime}$, est pris égal à $1 \mathrm{kPa}$. Les résultats expérimentaux montrent une forte réversibilité du chemin de déchargement isotrope $c_{s}$, l'incrément de l'indice des vides de obtenu, sur ce chemin, est donc élastique et peut s'exprimer comme $\mathrm{de}^{\mathrm{e}}=\frac{\mathrm{c}_{\mathrm{s}}}{2,3} \frac{\mathrm{d} \mathrm{p}^{\prime}}{\mathrm{p}^{\prime}}$, ce qui correspond à un incrément de déformation volumique élastique vérifiant (8-A).

$$
\mathrm{d} \varepsilon_{\mathrm{v}}^{\mathrm{e}}=\frac{\mathrm{c}_{\mathrm{s}}}{2,3\left(1+\mathrm{e}_{0}\right)} \frac{\mathrm{d} \mathrm{p}^{\prime}}{\mathrm{p}^{\prime}}
$$

\section{8}

\section{Description du modèle Cam-clay original}

Les modèles Cam-clay original et modifié, sont des lois élastoplastiques avec écrouissage isotrope, les deux écritures se distinguent par une expression différente de la loi d'écoulement (Roscoe et al., 1958; Schofield et Wroth, 1968). Ces modèles ont été développés à partir des bases théoriques de la plasticité, intégrant les résultats d'essais triaxiaux classiques réalisés sur des chemins isotropes, des chemins à contrainte latérale effective constante et à volume constant.

\section{Bifl}

\section{Bases théoriques et expérimentales}

Le modèle de Cam-clay est fondé sur quatre éléments principaux:

a) L'étude de l'essai triaxial sur chemin isotrope décrit par le système (7-A)

\section{b) Le concept de l'état critique}

Ce concept est une des bases fondamentales de l'écriture de la loi de Cam-clay original. Cela consiste à supposer qu'il existe un état critique (ou état de plasticité parfaite) pour lequel le matériau se caractérise par une variation de volume nulle. Celle-ci se produit pour une valeur constante du rapport $\frac{\mathrm{q}}{\mathrm{p}^{\prime}}=\frac{\mathrm{q}_{\mathrm{cnt}}}{\mathrm{p}_{\mathrm{crt}}^{\prime}}$, et un indice des vides critique $e_{\text {. }}$ La formulation complète de l'état critique se traduit par le système d'équations (1-B) du tableau II. Sa représentation qualitative dans l'espace (e, p', q) (Fig, 1-B) fait apparaître une courbe dont les projections dans les plans $\left(q, p^{\prime}\right)$ et $(e, q)$ nous renvoient aux formes habituelles (Biarez et Hicher, 1994). La projection dans le plan $\left(\mathrm{e}, \log \mathrm{p}^{\prime}\right)$ est une droite parallèle à la droite de chargement isotrope $c$. M est caractérisée expérimentalement dans le plan (q, p'), et correspond à l'angle de frottement mesuré à la plasticité parfaite. (2-B) exprime la relation entre $\emptyset_{\text {crit }}^{\prime}$ et $\mathrm{M}$. 


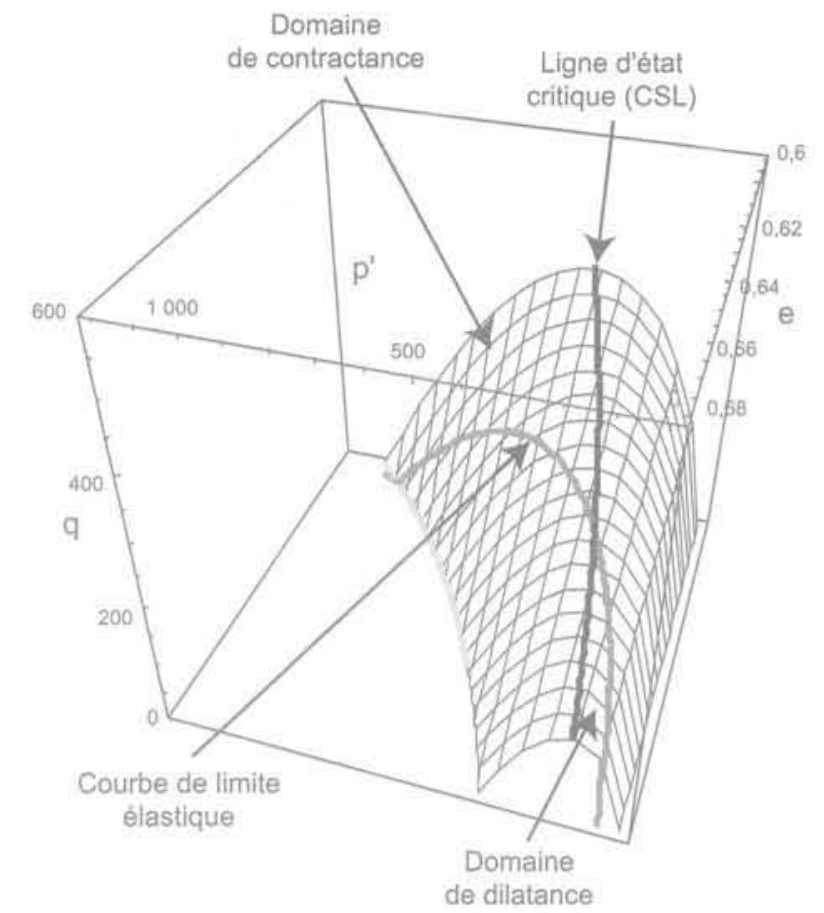

FG. 18 Domaine de contractance et de dilatance sur la surface de Roscoe.

Contraction and dilatancy domains on Roscoe surface.

\section{c) La loi d'écoulement plastique}

Elle découle de l'expression de l'incrément de dissipation plastique $\mathrm{dW}^{\mathrm{p}}$ exprimé dans le cas d'un chemin triaxial axisymétrique (3-B du tableau II). L'hypothèse fondamentale de la loi consiste à considérer que l'expression de $\mathrm{dW}^{\mathrm{p}}$ calculée à l'état critique $\left(\mathrm{dW}^{\mathrm{p}}=\right.$
$\mathrm{Mp}^{\prime} \mathrm{d} \bar{\varepsilon}^{\mathrm{P}}$ ), garde toujours la même expression tout au long du trajet de chargement. Il en découle la loi d'écoulement exprimée par (4-B) du tableau II.

\section{d) La règle de normalité}

La quatrième base fondamentale utilise la loi de normalité ((5-B) du tableau II) afin de formuler l'expression de fonction du potentiel $\mathrm{g}\left(\mathrm{q}, \mathrm{p}^{\prime}\right)$. La loi d'écoulement permet de déduire une équation différentielle puis sa résolution ((6-B) du tableau II). p' étant la contrainte de consolidation isotrope, liée à l'indice des vides " plastique $x$ e, et donc à la déformation volumique plastique, celle-ci apparaît comme paramètre d'écrouissage (Zienkiewicz et Naylor, 1972). Comme le modèle Camclay suppose une loi associée, nous avons égalité entre la fonction de charge f et la fonction potentiel g, la constante d'intégration peut également être calculée à partir d'un point de l'état critique $\left(\mathrm{e}_{\mathrm{crit}} \mathrm{p}_{\mathrm{crit}}^{\prime} \mathrm{q}_{\mathrm{cta}}\right)$ ) ce qui permet d'obtenir les expressions (7-B) (tableau II) des fonctions $f$ et $g$.

\section{B:z \\ Modèle Cam-clay dans l'espace (e, p', q) (Fig. 2-B)}

Avant chargement triaxial $(\mathrm{q}=0)$ l'état initial se trouve dans le plan $\left(\mathrm{e}, \mathrm{p}^{\prime}\right)$, puis selon la valeur du degré de surconsolidation, l'écoulement plastique est activé directement (cas des argiles normalement consolidées) ou après une première phase élastique (cas des argiles surconsolidées). L'écoulement plastique activé, les chemins triaxiaux du modèle Cam-clay évoluent sur la surface de Roscoe et s'arrêtent dès qu'ils atteignent la ligne d'état critique. Quand le comportement est élastique, le chemin se trouve à l'intérieur de la surface de Roscoe, et évolue sur une surface incurvée équivalente à la surface expérimentale $\mathrm{C}_{\mathrm{s}}^{3 \mathrm{~d}}$, décrite dans le texte et

TABLEAUII Modèle Cam-clay original. Cam-clay model.

\begin{tabular}{|c|c|c|c|}
\hline$\left\{\begin{array}{l}e=e_{\lambda}-c_{\varepsilon} \log \left(\frac{p^{\prime}}{p_{i}^{\prime}}\right) \\
e=e_{x}-c_{s} \log \left(\frac{p^{\prime}}{p_{i}^{\prime}}\right)\end{array}\right.$ & $\begin{array}{c}(7-A) \\
\text { (voir annexe } A)\end{array}$ & $\frac{\mathrm{d} \varepsilon_{i}^{p}}{\mathrm{~d} \bar{\varepsilon}^{p}}=\mathrm{M}-\frac{\mathrm{q}}{\mathrm{p}^{\prime}}$ & $(4-B)$ \\
\hline $\begin{aligned} & d \varepsilon_{v}^{p}=0 \\
& \frac{q_{c r i t}}{p_{c r i t}^{\prime}}=M \\
e_{c}= & \Gamma-c_{s} \log \left(\frac{p_{c r i t}^{\prime}}{p_{1}^{\prime}}\right)\end{aligned}$ & $(1-B)$ & $d \underline{\underline{\varepsilon}}^{p}=\lambda \frac{\partial g(\underline{\sigma})}{\partial \underline{\underline{\sigma}}}$ & (5-B) \\
\hline$\phi_{c r h}^{\prime}=\arcsin \left(\frac{3 M}{6+M}\right)$ & $(2-B)$ & $\begin{array}{c}\mathrm{M}-\frac{\mathrm{q}}{\mathrm{p}^{\prime}}=-\frac{\mathrm{dq}}{\mathrm{dp} \mathrm{p}^{\prime}} \\
\frac{\mathrm{q}}{\mathrm{p}^{\prime}}=-\mathrm{M} \ln \left(\mathrm{p}^{\prime}\right)+\mathrm{const}\end{array}$ & $(6-B)$ \\
\hline $\begin{aligned} \mathrm{dW}^{p} & =\sigma^{\prime} \mathrm{d} \varepsilon^{p} \\
& =\sigma^{\prime} \mathrm{d} \varepsilon^{p}+2 \sigma^{\prime} \mathrm{d} \varepsilon^{p} \\
& =q \mathrm{~d} \bar{\varepsilon}^{p}+p^{\prime} \mathrm{d} \varepsilon_{\mathrm{y}}^{p}\end{aligned}$ & (3-B) & $\begin{array}{c}g\left(q, p^{\prime}\right)=q+M p^{\prime} \ln \frac{p^{\prime}}{p_{i c}^{\prime}} \\
f\left(q, p^{\prime}, \varepsilon_{v}^{p}\right)=\frac{q}{M p^{\prime}}+\ln \left(\frac{p^{\prime}}{p_{c}^{\prime}}\right)-1\end{array}$ & $(7-B)$ \\
\hline $\mathrm{d} \varepsilon^{\mathrm{c}}=\frac{\mathrm{C}_{\mathrm{s}}}{2,3} \frac{\mathrm{dp} \mathrm{p}^{\prime}}{\mathrm{p}^{\prime}}$ & & $\frac{\mathrm{c}_{\mathrm{s}}}{3\left(1+\mathrm{e}_{0}\right)} \frac{\mathrm{dp}}{\mathrm{p}^{\prime}}$ & $(8-B)$ \\
\hline
\end{tabular}




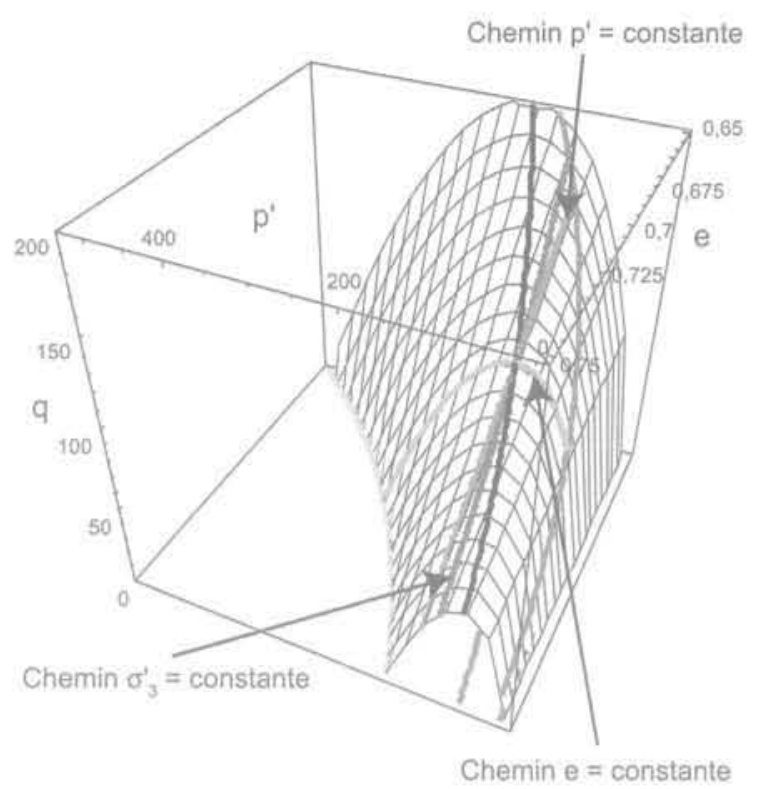

FIG. 28 Évolution de différents chemins du modèle Cam Clay dans (e, p', q) $\left(\mathrm{p}^{\prime}=\right.$ constante $)$ $\left(\sigma_{3}^{\prime}=\right.$ constante $)(\mathrm{e}=$ constante $)$.

Evolution of different Cam Clay Model paths in $\left(e, p^{\prime}, q\right)\left(p^{\prime}=\right.$ constante $)\left(\sigma_{3}^{\prime}=\right.$ constante $)$ $(\mathrm{e}=$ constante $)$.

représentée en figure 9, les relations (8-B du tableau II) donnent l'expression de la déformation volumique élastique. Lorsque ce chemin atteint la surface de Roscoe, l'écoulement plastique débute et suivant la position du point initial sur la courbe de déchargement isotrope, le processus d'écoulement évoluera différemment. Nous distinguerons l'argile très surconsolidée (OCR > e) et l'argile faiblement surconsolidée $(\mathrm{OCR}<\mathrm{e})$. La valeur $\mathrm{OCR}=$ e correspond à l'intersection entre la courbe de déchargement isotrope et la projection de la ligne d'état critique sur le plan (e, p').

Pour une courbe $\mathrm{c}_{\mathrm{s}}$ donnée, la courbe obtenue par l'intersection entre la surface élastique et la surface de Roscoe, est le lieu des points où l'écoulement plastique va se produire. La ligne d'état critique et cette courbe de limite élastique fixent sur la surface de Roscoe les domaines de dilatance caractérisant l'écoulement des argiles très surconsolidées (OCR > e), et de contractance caractérisant l'écoulement des argiles faiblement surconsolidées $(\mathrm{OCR}<\mathrm{e})$.

Le fonctionnement du modèle original est représenté, par la figure 2-B à partir des résultats d'essai triaxiaux dans (e, p', q). L'argile est sous trois chemins de chargement, le chemin à contrainte moyenne effective constante $\left(\mathrm{p}^{\prime}=\right.$ constante), le chemin à contrainte latérale effective constante $\left(\sigma_{3}^{\prime}=\right.$ constante) et le chemin non drainé(e = constante). Les paramètres du modèle sont déduits des résultats d'essais triaxiaux (Hattab, 1995). On a : $M=1,05, c_{s}=0,191, c_{s}=0,066$, $\mathrm{e}_{2}=1,17, \mathrm{p}_{\mathrm{co}}^{\prime}=1000 \mathrm{kPa}$. La modélisation de Cam-clay propose une continuité des chemins $\left(\left(\sigma_{3}^{\prime}=\right.\right.$ constante $)$, ( $\mathrm{p}^{\prime}=$ constante), $(\mathrm{e}=$ constante $\left.)\right)$ sur la surface de charge, ainsi qu'une convergence vers un point unique situé sur la courbe d'état critique. Par conséquent, pour un point donné sur la courbe d'état critique dans l'espace ( $p$ ', e, q), nous pouvons trouver une infinité de chemins menant à ce point.

\section{Lois d'écoulement plastique}

Les lois d'écoulement plastique, dans les modèles de comportement, sont souvent basées sur deux lois fondamentales rée dans le modèle Cam-clay et proposée pour les argiles (Roscoe et al., 1958; Schofield et Wroth, 1968), et celle de Rowe, proposée pour les sables (Rowe, 1962). Roscoe, en se basant sur le concept d'état critique (annexe B), propose une loi linéaire (4-B) entre le rapport des contraintes $\frac{q}{p^{\prime}}$ et le rapport des incréments des déformations plastiques $\frac{d \varepsilon_{v}^{p}}{d \bar{\varepsilon}^{p}}$.

La loi de Rowe (1-C), basée sur une linéarité entre le rapport de contraintes $\frac{\sigma_{1}^{\prime}}{\sigma_{3}^{\prime}}$ et le taux de dilatance $1-\frac{d \varepsilon_{v}^{p}}{d \varepsilon_{1}^{p}}$, a été établie afin de décrire l'écoulement plastique dans les sables.

$$
\frac{\sigma_{1}^{\prime}}{\sigma_{3}^{t}}=K\left(1-\frac{d \varepsilon_{v}^{p}}{d \varepsilon_{1}^{p}}\right)
$$

Faisant l'hypothèse que le paramètre $\mathrm{K}$ s'exprime en fonction de l'angle de frottement interne $\varnothing^{p p}$, calculé à la plasticité parfaite, on a: $K=\operatorname{tg}^{2}(\Pi / 4+\phi p p / 2)$. Nous pouvons alors établir une relation entre $\mathrm{M}$ et $\mathrm{K}$, soit, $\mathrm{K}=\frac{3+2 \mathrm{M}}{3-\mathrm{M}}$. La loi (1-C) se traduit, donc, par la relation

(2-C) lorsqu'elle est exprimée en fonction de $\frac{q}{p^{\prime}}$ et $\frac{d \varepsilon_{v}^{p}}{d \bar{\varepsilon}^{p}}$.

$$
\frac{d \varepsilon_{v}^{p}}{d \bar{\varepsilon}^{p}}=\frac{1}{1+M / 3\left(1-2 / 3 \frac{q}{p^{\prime}}\right)} \cdot\left(M-\frac{q}{p^{\prime}}\right)
$$

